\title{
Improved formulae for X-ray Reflectivity
}

\author{
Yoshikazu Fujii* \\ Kobe University, Nada-ku, Kobe 657-8501 Japan \\ * Corresponding author: Fax: 81-78-803-6116, e-mail: fujiiyos@kobe-u.ac.jp
}

\begin{abstract}
In the conventional X-ray reflectivity (XRR) analysis, the reflectivity is calculated based on the Parratt formalism, accounting for the effect of roughness by the theory of Nevot-Croce. However, the calculated results of the XRR done in this way often showed strange results where the amplitude of the oscillation due to the interference effects would increase for a rougher surface. For the solution to this problem, we have developed an improved formalism in which the effects of the roughness-induced diffuse scattering are included correctly. In this paper, for deriving more accurate formalism of XRR, we introduce the effective roughness depending on the angle of incidence X-ray in XRR measurement. The new improved XRR formalism derives more accurate surface and interface roughness with depending on the size of coherent $\mathrm{X}$-rays probing area, and derives the roughness correlation function and the lateral correlation length.
\end{abstract}

Key words: X ray reflectivity, surface and interface roughness, multilayer surfaces

\section{INTRODUCTION}

For structure investigations of multilayered thin film, X-ray reflectivity (XRR) is a powerful tool.[1-11] In the conventional XRR analysis, the reflectivity is calculated based on the Parratt formalism, accounting for the effect of roughness by the theory of Nevot-Croce.[1,2] In the conventional $x$-ray reflectivity analysis, the reflectivity $R$ from a multilayer consisting of $N$ layers with a flat surface and flat interfaces is calculated based on the Parratt formalism[1] as the following equations;

$$
\begin{aligned}
& R=\left|R_{0,1}\right|^{2}, R_{j-1, j}=\frac{\Psi_{j-1, j}+R_{j, j+1}}{1-\Psi_{j, j-1} R_{j, j+1}} \exp \left(2 i k_{j-1, z} h_{j-1}\right), \\
& R_{N, N+1}=0, \quad k_{j, z}=k \sqrt{n_{j}{ }^{2}-\cos ^{2} \theta},
\end{aligned}
$$

where $h_{j}$ is the thickness of $j$-th layer, $k_{\mathrm{j}, z}$ is the $z$-direction component of the wave vector in the $j$-th layer, $k=2 \pi / \lambda, \lambda$; wave length, $\theta$ is glancing angle of incidence, $n_{j}$ is the refractive index of the $j$-th layer, $n_{0}=$ 1. $\Psi_{j-1, j}$ is the Fresnel coefficients for reflection at the interface between $(j-1)$-th and $j$-th layers as

$$
\Psi_{j-1, j}=\frac{k_{j-1, z}-k_{j, z}}{k_{j-1, z}+k_{j, z}}, \quad \Psi_{j, j-1}=-\Psi_{j-1, j} .
$$

When the surface and interface have roughness, the Fresnel coefficient for reflection from rough surface and rough interfaces is reduced by the roughness. The effect of such roughness was taken into account only through the effect of the changes in density of the medium in a vertical direction to the surface and interface. With the use of relevant roughness parameters like the rms roughness $\sigma_{j-1}, j$ between $(j-1)$-th and $j$-th layers, the reduced Fresnel reflection coefficient $\Psi_{j-1, j}$ is derived by Nevot-Croce [2] and well known as showed

$$
\begin{aligned}
\Psi_{j-1, j} & =\frac{k_{j-1, z}-k_{j, z}}{k_{j-1, z}+k_{j, z}} Q_{j-1, j}, \quad \Psi_{j, j-1}=-\Psi_{j-1, j}, \\
Q_{j-1, j} & =\exp \left(-2 k_{j-1, z} k_{j, z} \sigma_{j-1, j}{ }^{2}\right),
\end{aligned}
$$

where $Q_{j-1, j}$ is the reduce factor due to the roughness. In this way, the conventional XRR formulae was derived with based on the Parratt formalism, accounting for the effect of roughness by the theory of Nevot-Croce.[1,2] Figure 1 shows the reflectivity from a tungsten-covered silicon wafer calculated by the conventional XRR formulae.

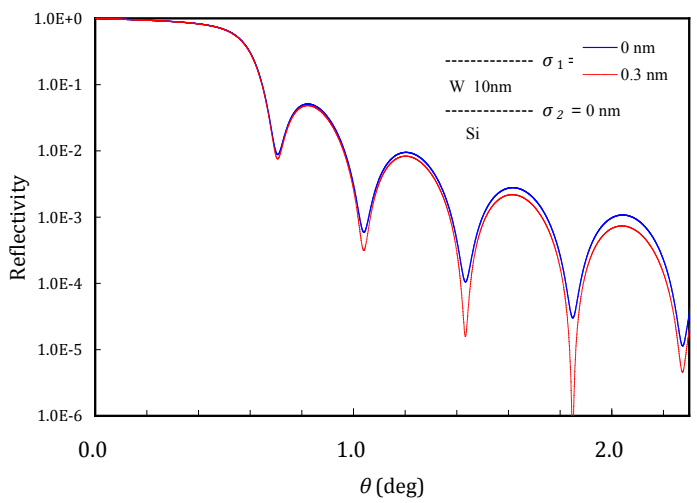

Fig. 1. X-ray reflectivity from a silicon wafer covered with a thin $(10 \mathrm{~nm})$ tungsten film calculated by the conventional XRR formulae. Solid line shows the case of a flat surface. Dashed line shows the case of a surface with a surface roughness of $0.3 \mathrm{~nm}$.

The ratio of the oscillation amplitude to the value of the reflectivity from a surface with a surface roughness of $0.3 \mathrm{~nm}$ (dashed line) does not decrease near an angle of incidence of $1.8^{\circ}$ 
but increase. We consider the origin of the above strange behavior was attributed to the fact that the diffuse scattering at the rough interface was not correctly taken into account in the XRR formalism based on the Parratt formalism with the use of the Nevot and Croce approach to account for roughness.[1,2]

Then we have developed a new formalism in which the effects of the roughness-induced diffuse scattering are included correctly.[12-14] The developed new accurate reflectivity equation of $R_{\mathrm{j}-1, \mathrm{j}}$ and $R_{\mathrm{j}, \mathrm{j}+1}$ is showed as follows,

$R_{j-1, j}=\frac{\Psi_{j-1, j}+\left(\Phi_{j-1, j} \Phi_{j, j-1}-\Psi_{j-1, j} \Psi_{j, j-1}\right) R_{j, j+1}}{1-\Psi_{j, j-1} R_{j, j+1}} \exp \left(2 i k_{j-1, z} h_{j-1}\right)$,

where $\Psi_{j-1, j}$ and $\Phi_{j-1, j}$ are the Fresnel coefficients for reflection and refraction, respectively, at the interface between the $(j-1)$ th layer and the $j$-th layer. In the new improved formulae for the $x$-ray reflectivity, the well-known reduced Fresnel coefficients of eqs. (3) and (4) for reflection is applied to the Fresnel coefficients $\Psi_{j-1, j}$ for reflection at rough interface. While an accurate analytical formula for the Fresnel coefficients $\Phi_{j-1, j}$ for refraction at rough interface is not available. There are several approximations proposed so far and all these results can be written by including any parameters depend on the proposed approximations [3-6] as showed,

$$
\begin{aligned}
& \Phi_{j-1, j}=\frac{2 k_{j-1, z}}{k_{j-1, z}+k_{j, z}} P_{j-1, j}, \quad \Phi_{j, j-1}=\Phi_{j-1, j} \frac{k_{j, z}}{k_{j-1, z}}, \\
& P_{j-1, j}=\exp \left\{-\left[C_{1}\left(k_{j-1, z}-k_{j, z}\right)^{2}+C_{2} k_{j-1, z} k_{j, z}\right] \sigma_{j-1, j}{ }^{2}\right\},
\end{aligned}
$$

where $P_{j-1, j}$ is the reduce factor due to the roughness, and parameters $C_{1}, C_{2}$ depend on the proposed approximation.[3-6] In the previous work, we chose $C_{1}=2$ and $C_{2}=0$ as the most appropriate approximation. [14] Based on the above considerations, we again calculated the X-ray reflectivity for the W/Si system, but now considered the effect of attenuation in the refracted $\mathrm{X}$ rays by diffuse scattering resulting from surface roughness. Figure 2 shows the reflectivity by the new improved XRR formulae.

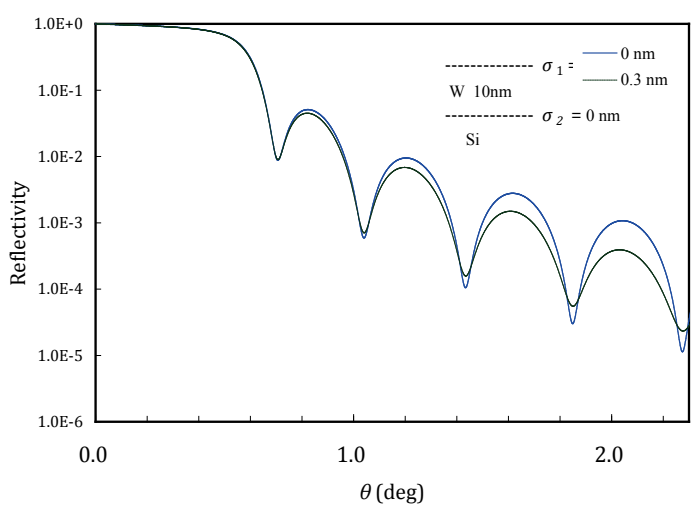

Fig. 2. X-ray reflectivity from a silicon wafer covered with a thin $(10 \mathrm{~nm})$ tungsten film calculated by the new improved formulae that considered diffuse scattering. Solid line shows the case of a flat surface. Dashed line shows the case of a surface with a surface roughness of $0.3 \mathrm{~nm}$.
The amplitude of the oscillation in the reflectivity curve from a surface with a surface roughness of $0.3 \mathrm{~nm}$ (dashed line) has reduced in Figure 2. These results are now physically reasonable. The strange results seen in Figure 1 have disappeared in Figure 2. It seems natural that the effect of interference does decrease at a rough surface and interface, because the amount of coherent $\mathrm{X}$ rays should reduce due to diffuse scattering. The reflectivity by this improved formalism gives a physically reasonable result. However the reduced Fresnel coefficients in the reflectivity equation needs further research and refine.

For deriving more accurate formalism of XRR, we tried to compare the measurements of the surface roughness of the same sample by atomic force microscopy (AFM) and XRR. The results of analysis showed that the effective roughness measured by XRR might depend on the angle of incidence.[15] This suggested that the value of roughness measured by the XRR might change by an incidence angle of X-ray, and changed very much at small glancing incidence angle. This suggested that the effective roughness depending on an incidence angle in XRR calculation should be assumed.

In this paper, we show the improved formulae of XRR which derives more accurate surface and interface roughness with depending on the size of coherent X-rays probing area.

\section{COMPARISON WITH ATOMIC FORCE \\ MICROSCOPY AND X-RAY REFLECTIVITY}

In the comparison with atomic force microscopy (AFM) and XRR on the measurements of the surface roughness,[15] we examined a sample of silicon wafers having a thin $\mathrm{SiO}_{2}$ layer, which was prepared by vacuum deposition of an additional $\mathrm{SiO}_{2}$ layer of about $2 \mathrm{~nm}$ on the $\mathrm{SiO}_{2}$ layer of about $5 \mathrm{~nm}$ prepared by thermal oxidizing of a $\mathrm{Si}(001)$ wafer.

By the AFM observations, we found that the r.m.s. roughness $\sigma_{\mathrm{s}}$ at the area of $1 \times 1 \mu \mathrm{m}^{2}$ of the $\mathrm{SiO}_{2}$ surface was about $0.17 \mathrm{~nm}$, and those at the area of $10 \times 10 \mu \mathrm{m}^{2}$ were about $0.24 \mathrm{~nm}$. The surface roughness by AFM observation depended on the observation area.[15]

$\mathrm{X}$-ray reflectivity measurements were performed using a $\mathrm{Cu}-\mathrm{K} \alpha \mathrm{X}$-ray beam from a $3 \mathrm{~kW}$ rotating-anode source. The beam size of the X-ray was about $2 \mathrm{~mm}$ (perpendicular to the reflection plane) $\times 0.05 \mathrm{~mm}$ (parallel to the reflection plane). In comparison with AFM and XRR, the surface roughness by XRR was different from the AFM observation. The surface roughness estimated from AFM observation showed small value with those of XRR, and showed smaller at the area of $1 \times 1 \mu \mathrm{m}^{2}$ than at the area of $10 \times 10 \mu \mathrm{m}^{2}$. This suggested that the value of roughness measured by the measurement range might be different in the XRR measurements. And the XRR measurement range changed by an incidence angle, and changed very much at small glancing incidence angle. This suggested that the effective roughness depending on an incidence angle in XRR calculation should be assumed.

After analyzing the XRR results, the thickness of the $\mathrm{SiO}_{2}$ layer was determined to be $7.8 \mathrm{~nm}$, the interface 
roughness $\sigma_{\mathrm{i}}$ was determined to be $0.42 \mathrm{~nm}$. Using these values $\left(\sigma_{\mathrm{i}}=0.42 \mathrm{~nm}\right.$ and the thickness $\left.7.8 \mathrm{~nm}\right)$ the reflectivity was calculated with various values of $\sigma_{\mathrm{s}}$. Figure 3 shows the comparison between the calculated and experimental results. None of the calculated results can reproduce the experimental one. At $\theta_{\mathrm{i}}>1.0^{\circ}$ the calculated result for $\sigma_{\mathrm{s}}=0.54 \mathrm{~nm}$ agrees with the experimental one while the calculated result deviates from the experimental one at smaller $\theta_{\mathrm{i}}$. On the other hand, the calculated result for $\sigma_{\mathrm{s}}=1.08 \mathrm{~nm}$ agrees with the experimental one at smaller $\theta_{\mathrm{i}}$ but it deviates seriously with increasing $\theta_{\mathrm{i}}$. A possible explanation of the present discrepancy might be that the effective surface roughness measured by XRR depends on the size of the effective probing area on the surface, which is proportional to $1 / \sin \theta_{\mathrm{i}}$. In general, the surface roughness increases with increasing size of the probing area. As a result, the effective roughness observed at smaller $\theta_{\mathrm{i}}$ is larger than that at larger $\theta_{\mathrm{i}}$ in accordance with the present result. Such a $\theta_{\mathrm{i}}$-dependence of the effective roughness in XRR has been usually neglected. The present result, however, indicates that it should be taken into account of which the effective roughness depends on the probing area size of the coherent X-ray.

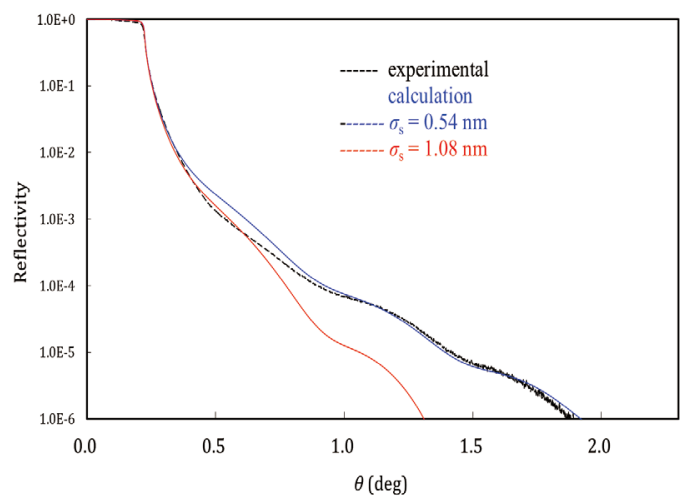

Fig. 3. X-ray reflectivity from $\mathrm{SiO} 2 / \mathrm{Si}$. The experimental result (thick dashed curve) is compared with the calculated ones for various $\sigma_{s}$ (thin curves).

\section{IMPROVED FORMALISM OF X-RAY \\ REFLECTIVITY}

Now we consider about the reduce factor due to the roughness again. Previous work, $[4,6] \mathrm{X}$-ray scattering from rough surface is studied, and the effect of the roughness is explained as,

$$
\begin{aligned}
& Q_{j-1, j}=\frac{\iint_{S_{0}} e^{-k_{j-1, k} k_{j, z} g(x, y)} e^{-i\left(q_{x} x+q_{y}, y\right)} d x d y}{\iint_{S_{0}} e^{-i\left(q_{x} x+q_{y}, y\right)} d x d y}, q_{x}=k_{j, x}-k_{j, x}, q_{y}=k_{j, y}-k_{j, y}, \\
& P_{j-1, j}=\frac{\iint_{S_{0}} e^{-1 / 4\left(k_{j-1, x}-k_{j, z}\right)^{2} g(x, y)} e^{-i\left(q_{x} x+q_{y} y\right)} d x d y}{\iint_{S_{x}} e^{-i\left(q_{x} x+q_{y}, y\right)} d x d y} q_{x=k_{j-1, x}-k_{j, x}, q_{y}=k_{j-1, y}-k_{j, y}},
\end{aligned}
$$

where $g(x, y)=\left\langle\left\{z\left(x^{\prime}+x, y^{\prime}+y\right)-z\left(x^{\prime}, y^{\prime}\right)\right\}^{2}\right\rangle$ is the square average of the height of the interface at $\left(x^{\prime}+x, y^{\prime}+y\right)$ separated by $(x, y)$ from $\left(x^{\prime}, y^{\prime}\right)$. In the reflected $\mathrm{X}$-ray and the refracted X-ray, $q_{x}=q_{y}=0$, and the scattering plane, $x-z$ plane, is considered in the analysis on X-ray reflectivity. Then the reduce factor $Q_{j-1, j}$ and $P_{j-1, j}$ are shown as,

$$
\begin{aligned}
& Q_{j-1, j}=\frac{1}{L_{x}} \int_{L_{x}} e^{-k_{j-1,2} k_{j, z} g(x)} d x \\
& P_{j-1, j}=\frac{1}{L_{x}} \int_{L_{x}} e^{-1 / 4\left(k_{j-1, z}-k_{j, z}\right)^{2} g(x)} d x,
\end{aligned}
$$

where $L_{x}$ is the length of the probing area of coherent $\mathrm{X}$-ray. The square average $g(x)$ of the height of the interface is related to the roughness correlation function $C(x)$ as, $g(x)=2 \sigma^{2}-2 C(x)$

Following Shinha et al.,[4] the roughness correlation function $C(x)$ of a fractal surface has the form as,

$$
g(x)=2 \sigma^{2}\left[1-\exp \left\{-\left(\frac{|x|}{\xi}\right)^{2 H}\right\}\right],
$$

where Hurst parameter $H(0<H \leq 1)$ is connected to its fractal dimension, and the lateral correlation length $\xi$ acts as a cutoff length for the fractal behavior of the surface. Then the reduce factor shows as the following;

$$
\begin{aligned}
& Q_{j-1, j}=\int_{0}^{1} \exp \left(-2 k_{j-1,2} k_{j, z} \sigma_{j-1, j}^{2}\left[1-\exp \left\{-\left(\frac{L_{x} x}{\xi_{j-1, j}}\right)^{2 H}\right\}\right]\right) d x, \\
& P_{j-1, j}=\int_{0}^{1} \exp \left(-\frac{1}{2}\left(k_{j-1,2}-k_{j, z}\right)^{2} \sigma_{j-1, j}{ }^{2}\left[1-\exp \left\{-\left(\frac{L_{x} x}{\xi_{j-1, j}}\right)^{2 H}\right\}\right]\right) d x,
\end{aligned}
$$

when $\xi<<L_{x}, g(x)$ becomes $2 \sigma^{2}$, and the reduce factor $Q_{j-1, j}$ becomes Eq.(4). Now we assumed that the roughness include the part without lateral correlation, i.e., $\sigma$ ' when $\xi=0$. Then the square average $g(x)$ of the height of the interface becomes;

$$
g(x)=2 \sigma^{2}\left[1-\exp \left\{-\left(\frac{|x|}{\xi}\right)^{2 H}\right\}\right]+2 \sigma^{\prime^{2}} .
$$

Then the reduce factor shows as the following;

$$
\begin{aligned}
& Q_{j-1, j}=\int_{0}^{1} \exp \left(-2 k_{j-1,2} k_{j, 2}\left[\sigma^{\prime \prime}{ }_{j-1, j}{ }^{2}-\sigma_{j-1, j}{ }^{2} \exp \left\{-\left(\frac{L_{x} x}{\xi_{j-1, j}}\right)^{2 H}\right\}\right]\right) d x \\
& P_{j-1, j}=\int_{0}^{1} \exp \left(-\frac{1}{2}\left(k_{j-1,2}-k_{j, z}\right)^{2}\left[\sigma^{\prime \prime}{ }_{j-1, j}{ }^{2}-\sigma_{j-1, j}{ }^{2} \exp \left\{-\left(\frac{L_{x} x}{\xi_{j-1, j}}\right)^{2 H}\right\}\right] d x\right.
\end{aligned}
$$

And we assume more approximation on integral of double exponential function that replace a value of the integral calculus with the value of the integrand at $x=1$. Then the reduce factor shows as the following;

$$
\begin{aligned}
& Q_{j-1, j}=\exp \left(-2 k_{j-1, z} k_{j, z}\left[\sigma^{\prime \prime}{ }_{j-1, j}{ }^{2}-\sigma_{j-1, j}{ }^{2} \exp \left\{-\left(\frac{L_{x}}{\xi_{j-1, j}}\right)^{2 H}\right\}\right]\right),(18) \\
& P_{j-1, j}=\exp \left(-\frac{1}{2}\left(k_{j-1,2}-k_{j, z}\right)^{2}\left[\sigma^{\prime \prime}{ }_{j-1, j}{ }^{2}-\sigma_{j-1, j}{ }^{2} \exp \left\{-\left(\frac{L_{x}}{\xi_{j-1, j}}\right)^{2 H}\right\}\right]\right) .
\end{aligned}
$$

Now we show the reduce factor with using the effective roughness $\sigma^{*}$ at the angle $\theta_{\mathrm{i}}$ of incident X-ray as,

$$
\begin{aligned}
& Q_{j-1, j}=\exp \left(-2 k_{j-1, z} k_{j, z} \sigma_{j-1, j}^{*}{ }^{2}\right), \\
& P_{j-1, j}=\exp \left(-\frac{1}{2}\left(k_{j-1, z}-k_{j, z}\right)^{2} \sigma_{j-1, j}^{*}{ }^{2}\right),
\end{aligned}
$$

where the effective roughness $\sigma^{*}$ can be defined approximately as,

$$
\sigma^{*}{ }_{j-1, j}{ }^{2}=\sigma^{\prime \prime}{ }_{j-1, j}{ }^{2}-\sigma_{j-1, j}{ }^{2} \exp \left\{-\left(\frac{L_{x}}{\xi_{j-1, j}}\right)^{2 H}\right\},
$$

Note that we implicitly assumed that $\xi$ is smaller than the coherence length $L_{x}$ of the radiation parallel to the surface. $L_{x}$ depends on the angle $\theta_{\mathrm{i}}$ of incident X-ray as,

$$
\frac{1}{L_{x}^{2}}=\frac{\sin ^{2} \theta_{i}}{L_{t}^{2}}+\frac{\cos ^{2} \theta_{i}}{L_{l}^{2}}
$$

where $L_{t}$ is transverse coherence length and $L_{l}$ is longitudinal coherence length of the incident $\mathrm{X}$ ray. 
Based on the above considerations, we again calculated the X-ray reflectivity for the $\mathrm{SiO}_{2} / \mathrm{Si}$ surface, but now considered the effective roughness $\sigma^{*}$ at the $\mathrm{X}$-ray incident angle $\theta_{\mathrm{i}}$. Figure 4 shows the calculated XRR with using the values; $\xi_{s}=2 \mu \mathrm{m}, L_{t}=10 \mathrm{~nm}$, and $L$ $=2 \mu \mathrm{m}$. The effective roughness is from about $1 \mathrm{~nm}$ to about $0.5 \mathrm{~nm}$ in the range of $\theta_{\mathrm{i}}$ in Fig. 4 . The calculated reflectivity shows good agreement with the experimental one in all range of measured $\theta_{\mathrm{i}}$.

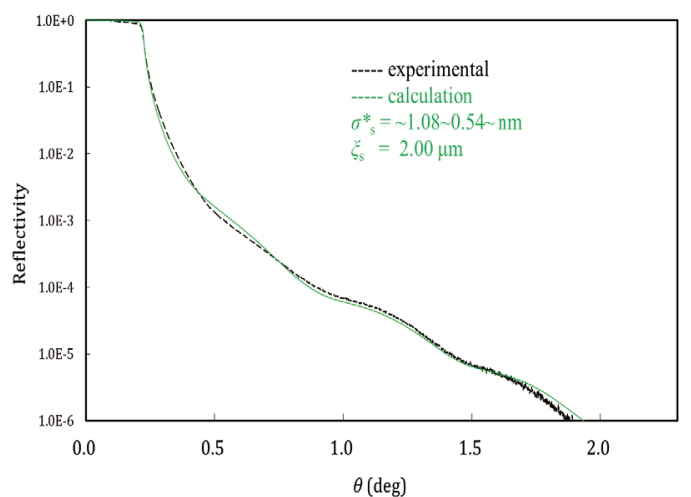

Fig. 4. X-ray reflectivity from $\mathrm{SiO} 2 / \mathrm{Si}$. The experimental result (thick dashed curve) is compared with the calculated ones for using the effective roughness depending on the X-ray incident angle $\theta_{\mathrm{i}}$ XRR is calculated with using the values $\left(\xi_{\mathrm{s}}=2 \mu \mathrm{m}, L_{\mathrm{t}}=10 \mathrm{~nm}\right.$, and $\left.L_{\mathrm{l}}=2 \mu \mathrm{m}\right)$.

\section{CONCLUSIONS}

In the XRR calculation, we introduced the effective roughness with depending on the incidence angle of X-ray. At the result, it is showed the new improved XRR formalism which derives more accurate surface and interface roughness with depending on the size of coherent X-rays probing area, and derives the roughness correlation function and the lateral correlation length.

\section{REFERENCES}

[1] Parratt, L. G. (1954). "Surface Studies of Solids by Total Reflection of X-Rays," Phys. Rev. 95, 359-369.

[2] Nevot, L. and Croce, P. (1980). "Caracterisation des surfaces par reflexion rasante de rayons $\mathrm{X}$. Application a l'etude du polissage de quelques verres silicates," Rev. Phys. Appl. 15, 761-779.

[3] Vidal, B and Vincent, P. (1984). "Metallic multilayers for x rays using classical thin-film theory," Applied Optics 23, 1794-1801.

[4] Sinha, S. K., Sirota, E. B., Garoff, S., and Stanley, H. B. (1988) "X-ray and neutron scattering from rough surfaces," Phys. Rev. B 38, 2297-2311.

[5] Holy, V., Kubena, J., Ohlidal, I., Lischka, K., and Plotz, W. (1993). "X-ray reflection from rough layered systems," Phys. Rev. B 47, 15896-15903.

[6] Boer, D. K. G. (1995). "X-ray reflection and transmission by rough surfaces," Phys. Rev. B 51, 5297-5305

[7] Daillant, J. and Gibaud, A. (Eds.) (1999). X-ray and Neutron Reflectivity, Principles and Applications (Berlin Springer).
[8] Holy, V., Pietsch, U., and Baumbach, T. (Eds.) (1999). High-Resolution X-ray Scattering from Thin Films and Multilayers (Berlin Springer).

[9] Fujii, Y., Nakayama, T., and Yoshida, K. (2004). "Roughness Estimation of Polycrystalline Iron Surface under High Temperature by Small Glancing Angle X-ray Scattering,” ISIJ International 44, 1549-1553. [10] Fujii, Y., Komai, T., and Ikeda, K. (2005)." Depth profiling of polycrystalline layers under a surface using x-ray diffraction at small glancing angle of incidence," Surf. Interface Anal. 37, 190-193.

[11] Sakurai, K. (Ed.) (2009). Introduction to X-ray Reflectivity (Kodansha Scientific).

[12] Fujii, Y. (2010). "Influence of surface roughness on near-surface depth analysis from X-ray reflectivity measurements," Surf. Interface Anal. 42, 1642-1645. [13] Fujii, Y. (2011). "Improved x-ray reflectivity calculations for rough surfaces and interfaces," Series: Materials Science and Engineering 24 012009-1-21. [14] Fujii, Y. (2013). "Improved x-ray reflectivity calculations on a multilayered surface," Powder Diffraction 28 (2), 100-104.

[15] Fujii, Y. (2014). "Improvement of surface and interface roughness estimation on X-ray reflectivity," Powder Diffraction 29 (3), 265-268.

(Received January 30, 2015; Accepted May 20, 2015) 